\title{
Catheter ablation of atrial fibrillation: the need for studies to assess the efficacy and safety of novel anticoagulants
}

\author{
Gerald V. Naccarelli • Mario D. Gonzalez
}

Received: 20 July 2012 / Accepted: 31 July 2012 / Published online: 28 September 2012

(C) Springer Science+Business Media, LLC 2012

For selected patients with atrial fibrillation (AF), catheter ablation is an important treatment alternative and has about a $70 \%$ success rate in carefully selected patients. Patients undergoing this procedure are at an increased risk of thromboembolic events during and following the ablation procedure. Bridging of anticoagulation, insertion of sheaths and catheters, left atrial endothelial damage, and atrial stunning may all lead to this increased thromboembolic risk. In order to minimize these thromboembolic events, anticoagulation, initiated as part of the procedure, increases bleeding complications including pericardial tamponade and vascular complications. Controlled data are not available to guide anticoagulation management of such patients during the peri-ablation period. Several years ago, we used to discontinue warfarin for several days before the procedure and the anticoagulation bridged with subcutaneous low molecular weight heparin (LMWH). The main limitation of this approach is that patients require LMWH after the procedure until the international normalized ratio (INR) becomes therapeutic. Excessive anticoagulation with LMWH and periods of subtherapeutic INR on warfarin post-procedure, have resulted in bleeding and thrombo-embolic complications [1]. More recently, in order to reduce these limitations, it has become more common to perform the ablation procedure without discontinuation of warfarin [2-4]. Several studies have demonstrated that uninterrupted warfarin is

\footnotetext{
G. V. Naccarelli $(\bowtie) \cdot$ M. D. Gonzalez

Electrophysiology Program, Heart and Vascular Institute,

Penn State University College of Medicine,

500 University Drive, Room H1511, P. O. Box 850, MC H047,

Hershey, PA 17033, USA

e-mail: gnaccarelli@hmc.psu.edu

M. D. Gonzalez

e-mail: mgonzalez@hmc.psu.edu
}

associated with fewer bleeding complications compared to LMWH bridging. With both approaches, unfractionated heparin is administered during the procedure and the dosing regimen is guided by a target activated clotting time (ACT) ranging from 225 to 450 . Heparin infusion is discontinued once all catheters have been removed and the sheaths are removed once the ACT is less than 200 s. Post-procedure LMWH is usually not required since the patient is on a therapeutic level of anticoagulation with warfarin. Anticoagulation is then continued for 3-6 months or indefinitely depending on the patient's risk factors.

The problem with the above protocols is that there is no gold standard based on carefully collected prospective controlled data and the FDA has never approved LMWH bridging as an indication for this type of perioperative use.

The commercial approval of dabigatran and rivaroxaban (and soon apixaban and edoxaban) has created a new anticoagulation challenge for AF patients undergoing catheter ablation procedures. Dabigatran has been commercially available for about 2 years and several reports have been published related to the safety and efficacy of using this drug during AF ablation procedures. Winkle et al. [5] demonstrated dabigatran to be safe and well tolerated when used after AF ablation (interrupted use prior to the ablation procedure) in a single-center study. The study included patients previously treated with warfarin, dabigatran, or aspirin. The post-procedure use of dabigatran was not associated with any major bleeding or thromboembolic events. However, three patients had to stop dabigatran due to dyspepsia, rash, and diarrhea. In this study, dabigatran was discontinued 36$60 \mathrm{~h}$ prior to the procedure based on renal function and restarted $22 \mathrm{~h}$ after the procedure (post-procedure LMW heparin was given). The target ACT during the procedure was $225 \mathrm{~s}$.

A larger multicenter study by Lakkireddy et al. [6] reported that periprocedural dabigatran use significantly 
increases the risk of bleeding or thromboembolic complications compared with uninterrupted warfarin therapy. Thromboembolic events occurred in $2.1 \%$ of dabigatran and $0 \%$ of warfarin patients $(p=0.19)$. Major bleeding trended higher $(14 \%)$ in the dabigatran versus $6 \%$ in the warfarin arm of the study (pNS). In this study, dabigatran was held on the morning of the procedure and restarted $3 \mathrm{~h}$ after the procedure and the target ACT was 300-400 s, using UF heparin, during the procedure. The results of this trial were puzzling in that both thromboembolic and bleeding complications appeared to be higher in the dabigatran arm of the study. These results caused many ablation centers to switch patients from dabigatran to warfarin prior to a scheduled ablation. However, in our center, we continue to keep patients on dabigatran or rivaroxaban pre- and post-procedure with a target intraprocedure ACT of 350 and we have not seen any significant complications using this protocol. In this issue of the Journal of Interventional Cardiac Electrophysiology, Snipelisky et al. [7], in a single-center study, demonstrated no difference between dabigatran- and warfarin-related embolic issues (no embolic events occurred in either arm of the study) or bleeding with a target ACT of at least $350 \mathrm{~s}$. This study suggested that there was more ACT variability in the dabigatran cohort of patients. This study adds to the published information on the safety and efficacy of using dabigatran in AF ablation procedures. Currently, there is no published data on the safety of rivaroxaban in this patient population but a prospective trial is planned by the sponsor.

Catheter ablation for $\mathrm{AF}$ is growing and the penetration of the market with new anticoagulants continues to grow. Thus, we need some answers and guidelines to help us through the proper anticoagulation regimen in this patient population. There are many moving parts to this issue: paroxysmal versus persistent AF, the CHADs2 score of the population studied, the technique used to obtain venous access (blind versus ultrasound guided), the aggressiveness and duration of the ablation procedure, target ACT during the procedure, use of intracardiac echocardiography to detect thrombus formation and pericardial effusion, use of externally irrigated electrode catheter for ablation, hemostasis after sheath removal, the time to hold an anticoagulant prior to the procedure and time to restart after the ablation, etc. It is our observation, that many complication occurring during and soon after the ablation (pericardial effusion, groin hematomas, arteriovenous fistulas, and pseudoaneurysms) are mainly related to technical factors, more than anticoagulation. Proper use of ultrasound to gain venous access, intracardiac echocardiography, externally irrigated catheters, close attention to impedance changes during radiofrequency energy delivery, and limits in the maximal power used have reduced the number of complications related to the procedure.

The number of major bleeds and embolic phenomenon from this procedure is small $(1-5 \%)$ so large-scale prospective trials really cannot be powered to answer all of these issues with certainty. Because of all of these confounding variables, a definitive study will never be designed. An AF ablation registry might add some useful information related to the safety and efficacy of the new anticoagulants related to $\mathrm{AF}$ ablation procedures. In the meantime, we need to continue to study various protocols related to this issue to guide physicians towards the proper use of these new drugs in the ablation population. All of the new anticoagulants should be studied in small but well-controlled studies to help guide the clinician in the use of these drugs in patients undergoing catheter ablation of atrial fibrillation.

\section{References}

1. Oral, H., Chugh, A., Ozaydin, M., Good, E., Fortino, J., Sankaran, S., Reich, S., Igic, P., Elmouchi, D., Tschopp, D., Wimmer, A., Dey, S., Crawford, T., Pelosi, F., Jr., Jongnarangsin, K., Bogun, F., \& Morady, F. (2006). Risk of thromboembolic events after percutaneous left atrial radiofrequency ablation of atrial fibrillation. Circulation, 114, 759-765.

2. Wazni, O. M., Beheiry, S., Fahmy, T., Barrett, C., Hao, S., Patel, D., Di Biase, L., Martin, D. O., Kanj, M., Arruda, M., Cummings, J., Schweikert, R., Saliba, W., \& Natale, A. (2007). Atrial fibrillation ablation in patients with therapeutic international normalized ratio: comparison of strategies of anticoagulation management in the periprocedural period. Circulation, 116, 2531-2534.

3. Hussein, A. A., Martin, D. O., Saliba, W., Patel, D., Karim, S., Batal, O., Banna, M., Williams-Andrews, M., Sherman, M., Kanj, M., Bhargava, M., Dresing, T., Callahan, T., Tchou, P., Di Biase, L., Beheiry, S., Lindsay, B., Natale, A., \& Wazni, O. (2009). Radiofrequency ablation of atrial fibrillation under therapeutic international normalized ratio: a safe and efficacious periprocedural anticoagulation strategy. Heart Rhythm, 6, 1425-1429.

4. Page, S. P., Siddiqui, M. S., Finlay, M., Hunter, R. J., Abrams, D. J., Dhinoja, M., Earley, M. J., Sporton, S. C., \& Schilling, R. J. (2011). Catheter ablation for atrial fibrillation on uninterrupted warfarin: can it be done without echo guidance. Journal of Cardiovascular Electrophysiology, 22, 265-270.

5. Winkle, R. A., Mead, R. H., Engel, G., Kong, M. H., \& Patrawala, R. A. (2012). The use of dabigatran immediately after atrial fibrillation ablation. Journal of Cardiovascular Electrophysiology, 23(3), 264-268.

6. Lakkireddy, D., Reddy, Y. M., Di Biase, L., Vanga, S. R., Santangeli, P., Swarup, V., Pimentel, R., et al. (2012). Feasibility and safety of dabigatran versus warfarin for periprocedural anticoagulation in patients undergoing radiofrequency ablation for atrial fibrillation: results from a multicenter prospective registry. Journal of the American College of Cardiology, 59(13), 1168-1174.

7. Snipelisky, D., Kauffman, C., Prussak, K., Johns, G., Venkatachalam, K., Kusumoto, F. (2012). A comparison of bleeding complications post ablation between dabigatran and warfarin. Journal of Interventional Cardiac Electrophysiology (in press). 